\title{
Antibiotic Resistance of Pathogens Causing Community-Acquired Pneumonia
}

${ }^{1}$ Charles Feldman and ${ }^{2}$ Ronald Anderson

${ }^{1}$ Division of Pulmonology, Department of Internal Medicine, Charlotte Maxeke Johannesburg Academic Hospital and Faculty of Health Sciences, University of the Witwatersrand, Johannesburg, South Africa and ${ }^{2}$ Medical Research Council Unit for Inflammation and Immunity, Department of Immunology, Faculty of Health Sciences, University of Pretoria and Tshwane Academic Division of the National Health Laboratory Service, South Africa.

\author{
ADDRESS AND CORRESPONDENCE \\ Professor Charles Feldman \\ Professor of Pulmonology \\ Division of Pulmonology \\ Department of Internal Medicine \\ University of the Witwatersrand \\ Medical School \\ 7 York Road \\ Johannesburg \\ South Africa \\ TEL + $2711488-3840$ \\ $\mathrm{FAX}+2711488-4675$ \\ e-mail charles.feldman@,wits.ac.za
}

\section{SUMMARY}

Community-acquired pneumonia remains an important cause of disease and death both in the developed and the developing worlds and therefore continues to have major medical impact. The mortality remains high despite the ready availability of potent antimicrobial 
agents to which the organisms are susceptible. However, management of these infections is potentially complicated by the emerging resistance of many of the common pathogens to the different classes of antibiotics that are usually prescribed. Furthermore, it is also being recognized that antibiotic resistance and/or treatment failures may occur not only through traditional microbial antibiotic resistance mechanisms, but also through less well-defined mechanisms, particularly those developed by the microbes in relation to their quorum sensing/biofilm machinery. Much recent research in this field has been focused on evaluating the clinical impact of antibiotic resistance on optimal antibiotic treatment and antimicrobial choices, as well as alternative strategies to deal with antibiotic resistance and/or treatment failures.

Key Words: antibiotic resistance, beta-lactam antibiotics, biofilm formation, community-acquired pneumonia, fluoroquinolones, macrolides, quorum sensing

\section{Introduction}

Community-acquired pneumonia (CAP) continues to be associated with significant morbidity and mortality in all regions of the world, with a high clinical and economic burden.${ }^{1-7}$ Short term mortality may be as high as $14 \%$ overall, while long term mortality reaches greater than $50 \%$ within 5 years. ${ }^{7}$ It is the only infectious disease consistently among the top ten causes of death worldwide and in many areas of the globe it is as high as the second most common cause. It is also the second leading cause for hospital admission, after childbirth. ${ }^{5}$ Treatment of these infections is potentially compromised by the rapid emergence of resistance of many of the usual pathogens to the most commonly prescribed antibiotics, including beta-lactams, macrolides/azalides and fluoroquinolones. ${ }^{5}$ 
Even multidrug resistant strains (commonly defined as resistance to 3 or more of the commonly used classes of agents) are begining to emerge. The current manuscript reviews important resistance issues among the most common CAP pathogens, highlighting both the conventional mechanisms of antibiotic resistance as well as resistance development associated with quorum sensing/biofilm formation, cigarette smoking and immunosuppression.

\section{Common pathogens associated with CAP}

The common pathogens causing CAP are very similar in many parts of the world, including the United States, Europe, Latin-America, Asia Pacific and sub-Saharan Africa, with a few exceptions. ${ }^{1-4,8-11}$ In most areas, the commonest isolate is Streptococccus pneumoniae (pneumococcus) and this holds true in cases treated in the outpatient, inpatient, or even the intensive care unit setting. ${ }^{12,13}$ It is therefore recommended that this pathogen should always be covered for with empiric antibiotic therapy. Other common pathogens are the so-called atypical pathogens, including Legionella pneumophila, Chlamydia pneumoniae and Mycoplasma pneumoniae, as well as the respiratory viruses. While these pathogens may cause CAP on their own they are often associated with other common bacterial pathogens, such as the pneumococcus, and this combination of microorganisms is more likely to be associated with a more severe pneumonia. Other pathogens that are commonly found include Staphylococcus aureus and some of the gram negative rods. The latter include Haemophilus influenzae and Moraxella catarrhalis, and in some areas, Enterobacteriaciae and occasionally others. It is clearly beyond the scope of this manuscript to describe completely all the issues in resistance with regard to all 
these pathogens, however, some of the more pertinent issues with regard to many of the more common pathogens are highlighted.

\section{Impact of antibiotic resistance on outcome}

While much is known about the prevalence, risk factors and mechanisms of antibiotic resistance, an important consideration is whether the presence of antimicrobial resistance alone is associated with adverse outcomes (e.g. death, treatment failure, persistence of infection). ${ }^{14-16}$ Less well studied, but certainly not of less concern, has been whether the presence of resistance has any clinical relevance in the treatment of common respiratory infections, such as the pneumococcus, treated with the common classes of antibiotics. ${ }^{17-19}$ In some infections, such as meningitis and otitis media, poor outcomes are much more likely to occur in association with antibiotic resistance; however, the evidence for this in CAP is considerably less clear. ${ }^{14,15,17,18}$ There are certainly multicentre studies from a number of regions of the world indicating that antimicrobial resistance has little impact on outcome. ${ }^{1,4}$ Nevertheless, it is clearly evident that antibiotic resistance is highly prevalent and increasing, ${ }^{18,20}$ and that antibiotic prescribing habits have changed as a consequence of perceived problems with resistance.

\section{Antimicrobial resistance among the common respiratory pathogens}

There has been considerable worldwide investigation into the prevalence, evolution, mechanisms and risk factors for antimicrobial resistance among the common respiratory pathogens, and particularly the pneumococcus, over a considerable period of time. ${ }^{14,18,20-}$

${ }^{24}$ With this has come the recognition not only that resistance pattens change over time, 
but also that there are significant geographical and regional differences in the patterns of resistance such that knowledge of local resistance patterns is essential in guiding the choice of initial empiric antibiotic therapy. ${ }^{12,18,22-24}$ Furthermore, more recent understanding of the pharmacokinetic and pharmacodynamic characteristics of the various antibiotics and their relationship to likely outcomes has helped inform decisions regarding antimicrobial therapy and its optimal dosing, particularly in the setting of antibiotic resistance..$^{17,19,25-27}$

\section{Streptococcus pneumoniae}

This microrganism has developed resistance to a myriad of antibiotics including the penicillins and cephalosporins (beta-lactams), macrolides and fluoroquinolones, and even multidrug resistance has begun to emerge. ${ }^{17,18,28-30}$ Little evidence existed for increased morbidity and/or mortality worldwide in patients with CAP infected with penicillin resistant pneumococci (based on existing breakpoint definitions of resistance) who were treated with penicillins and this lead to the Clinical and Laboratory Standards Institute (CLSI) revising the breakpoints for resistance in non-meningeal isolates in 2008 (susceptible defined as MIC levels $\leq 2 \mu \mathrm{g} / \mathrm{ml}$, intermediate resistance defined as MIC of $4 \mu \mathrm{g} / \mathrm{ml}$ and resistant as MIC of $8 \mu \mathrm{g} / \mathrm{ml}) .{ }^{11,14,29,30}$ This has resulted in the prevalence of penicillin resistance reported with the pneumococcus to be very much lower. ${ }^{31}$ The mechnism(s) of beta-lactam resistance in the pneumococcus are primarily related to alterations in the penicillin binding proteins. ${ }^{18}$ The latter are antibiotic target sites, which are integral to cell wall synthesis. ${ }^{18}$ It has been said that provided appropriate beta-lactam agents are used in adequate doses, current prevalences and levels of beta-lactam 
resistance among peumococci worldwide are such that resistance is unlikely to have any significant impact. ${ }^{17,18,26,28,29}$ Numerous studies attest to the ongoing success of betalactam therapy. ${ }^{26,32}$

In contrast to that with penicillin, macrolide resistance is a serious and highly prevalent problem worldwide. ${ }^{30}$ Two main mechanisms of macrolide resistance exist, namely, low level resistance (MIC 1-32 $\mu \mathrm{g} / \mathrm{ml}$ ), due to an active efflux pump mechanism (mef(A) gene), and high level resistance ( $\mathrm{MIC}>64 \mu \mathrm{g} / \mathrm{ml}$ ) due to a ribosomal methylation mechanism affecting the macrolide binding site (erm (B) gene) ${ }^{18,30}$ Different proportions of these two mechanisms exist in different parts of the world and in some areas, such as South Africa, both mechanisms may co-exist, translating overall into high-level resistance.

Although failures of macrolide therapy (clinical and/or microbiological) have been documented in the presence of macrolide resistance, these have been relatively few, and there is a rather poor correlation between macrolide resistance and treatment outcome. ${ }^{17,18,26,30,33-37}$ This has been described as the "in vivo-in vitro paradox", and there have been various suggestions forwarded to try and explain and understand this phenomenon. ${ }^{17,33,35,36}$ Nevertheless, it is commonly recommended that macrolides, as monotherapy for CAP, should be only used in areas where macrolide resistance is known to be low and in patients where risk factors for macrolide resistance are not present. ${ }^{17}$ Macrolides, as part of combination antibiotic therapy in more seriously ill patients, and to 
cover for so-called "atypical pathogens" in CAP, continue to play an important role in the management of patients with CAP. ${ }^{11,19,28}$

Resistance of the pneumococcus to fluoroquinolones is currently very uncommon, but is increasing worldwide. ${ }^{18,30,38}$ Fluoroquinolones inhibit bacterial DNA synthesis by interacting with the intracellular drug target, DNA gyrase and the enzymes topoisomerase IV. ${ }^{18}$ Resistance develops by mutations occurring in the quinolone resistance determining region (QRDR), involving gyrA and parC. In the case of low level resistance, mutations most commonly occur in parC, and these isolates test susceptible to the newer fluoroquinolones, whereas in high level resistance dual mutations occur affecting both parC and gyrA. ${ }^{18}$ Efflux pumps are also sometimes found, but the significance of this potential mechanism of resistance is unclear. ${ }^{18,39}$ In the presence of high level resistance with dual mutations, treatment failures with macrolides have been reported. ${ }^{18,37,40}$ However, what is less well known is that the presence of a first-step mutation in the QRDR, not translating into in vitro resistance, may evolve during treatment and result in the emergence of fluoroquinolone resistant strains during fluoroquinolone therapy. ${ }^{40}$ Unfortunately, the prevalence of these strains carrying singlestep mutations is unknown in many parts of the world.

Resistance to other antibiotic classes of antibiotics, a number of which are not commonly used or recommended for the treatment of CAP, has been described and is reviewed elsewhere. $^{18}$ 


\section{Mycoplasma pneumoniae}

Mycoplasmal organisms lack a cell wall and are therefore resistant to cell wall synthesis inhibitors, such as beta-lactams, glycopeptides and fosfomycin. However, in addition to tetracyclines and fluoroquinolones, macrolide antibiotics are effective therapeutic agents against the so-called "atypical pathogens". Macrolides are therefore recommended in many of the international CAP guidelines as first-line choice for the treatment of $M$. pneumoniae infections, which may be a cause of as many as $11-15 \%$ of cases of CAP. ${ }^{41}$ While macrolide resistance among M. pneumoniae has been emerging in children, particularly in countries such as Japan, more recently reports have begun to apppear, documenting the occurrence of CAP in adults due to macrolide resistant M. pneumoniae. In one of the first such case reports in adults, also from Japan a 28 year old lady was found to be infected with an isolate of M. pneumoniae with elevated MICs for the various macrolide/azalide/telithromycin antimicrobial agents, due to the same point mutation (A to $\mathrm{G}$ transition) at position 2063 in domain $\mathrm{V}$ of the $23 \mathrm{~S}$ rRNA gene that accounted for $>$ $90 \%$ of cases in the pediatric population in Japan. ${ }^{42}$ This mutation is associated with the highest level of resistance to the macrolide group of drugs. ${ }^{43}$ Further case studies have been reported and other mutations documented, although these are less common. ${ }^{44}$ Interestingly, none of the M. pneumoniae strains has been found to be resistant to either minocycline or the fluoroquinolones. Such strains have also subsequently been reported from other countries, including France, USA, Denmark and China ${ }^{43,44}$ More than $90 \%$ of isolates in China are resistant to erythromycin and azithromycin. ${ }^{45}$ The subject of mycoplasmal antibiotic susceptibility and resistance has been extensively reviewed elsewhere. ${ }^{45}$ The exact reason(s) for the emergence of these strains is unclear, but may 
relate to the use of low dose macrolides in chronic lung diseases, or possibly other reasons. ${ }^{44}$ The clinical course of these infections, especially the duration of fever and duration of cough in these patients, is prolonged and most cases had been treated with macrolides previously. ${ }^{43}$ In most situations, therapy in these cases has been changed to minocycline or a fluoroquinolone, with good clinical outcomes, although neither of these agents is ideal for children.

\section{Haemophilus influenzae and Moraxella catarrhalis}

H. influenzae is a common colonizer of the pharynx, even in healthy individuals, and may colonize the lower respiratory tract in patients with underlying respiratory disorders. ${ }^{46}$ While this microorganism may cause upper respiratory tract infections (URTI) (otitis media and sinusitis), and it plays a significant role in acute exacerbations (AE) of COPD, it may also be a cause of up to $15 \%$ of cases of CAP ${ }^{46}$ Since the 1970 s antibiotic resistance in this pathogen has been increasing. Similarly, M. catarrhalis has only more recently been recognized to be an important human pathogen, and is now recognized to be the second most important cause of AE of COPD, an important cause of URTI (sinusitis and otitis media) and also to cause up to $10 \%$ of cases CAP, especially at the extremes of age. ${ }^{46}$

Resistance to both these microorganisms is commonly due to due to emergence of betalactamase production. This was initially reported as TEM-1 type in the case of $H$. influenzae and later a novel new beta-lactamase was found that was termed ROB-1. ${ }^{47}$ There is also a suggestion that some isolates are also beta-lactamase resistant, but do not 
carry either TEM-1 or ROB-1 and therefore appear to have a novel beta-lactamase type. ${ }^{47}$ In the case of $M$. catarrhalis the beta-lactamases are most commonly those termed BRO1, BRO-2 or BRO- $3 .{ }^{46}$ The prevalence of resistance strains varies across the world. ${ }^{46,47}$ For example, in the PROTEKT Study (a surveillance study investigating antimicrobial susceptibility of respiratory pathogens around the world) there was a marked variation in the range of beta-lactamase production in the different regions in the case of $H$. influenzae (from $1.8 \%$ in Italy to $65 \%$ in South Korea), whereas $92 \%$ of all the isolates of M. catarrhalis in that study were found to be beta-lactamase positive. ${ }^{46}$

Other types and mechanisms of antibiotic resistance in the case of $H$. influenzae have been documented and extensively reviewed elsewhere. ${ }^{47}$ Strains of $H$. influenzae have also been documented to be resistant to ampicillin, but not due to production of betalactamases (beta-lactamase negative ampicillin resistance (BLNAR) strains), and the mechanism is thought to be due to alterations in the pencillin binding proteins (PBPs). ${ }^{47}$ These strains appear to be more common in Japan and France. ${ }^{47}$ Some strains have been found containing both these mechanisms of resistance (so-called BLPACR strains). ${ }^{47}$ It is rather worrying that in the PROTEKT study some $H$. influenzae isolates demonstrated intermediate resistance to macrolides, while most of the $M$. catarrhalis strains were sensitive to all the other antibiotics tested. ${ }^{46}$ Subsequently, a macrolide efflux pump mechanism was documented in H. Influenzae, although there is some debate about the clinical significance of this, since the isolates still have rather low MICs. ${ }^{47}$ Tetracycline resistance among these pathogens appears also to be due to an efflux pump and resistance to the fluoroquinolones is still very uncommon. ${ }^{47}$ Thus in the case of $H$. influenzae some 
caution needs to be exercised with the use of certain beta-lactams, as well as macrolide/azalides and tetracyclines, at least in certain areas. ${ }^{47}$

In the case of $M$. catarrhalis, beta-lactamase appears to be the main mechanism of resistance and so these pathogens remain susceptible to most of the other agents potentially used for CAP other than ampicillin/amoxicillin, including cephalosporins, macrolides/azalide, tetracycline and fluroquinolones. ${ }^{48,49}$

\section{Staphylococcus aureus}

S. aureus is an important cause of various severe infections in humans, including pneumonia. Methicillin-resistant isolates of S. aureus (MRSA) are uniformly resistant to all available penicillins and other beta-lactam agents. While infections with such microorganisms have classically been prevalent in the setting of the hospital environment, in other health-care facilities and in individuals with contact with such environments, since the 1990s there has been an explosion of patients with MRSA infections who do not have classical risk factors of contact with the hospital system and increasing recognition of the new strains of so-called community-associated MRSA (CAMRSA) infections. ${ }^{50-52}$ These infections have been extensively reviewed elsewhere. ${ }^{52,53}$ Such pathogens seem to be more common in the USA than in Europe (11). CA-MRSA isolates are resistant to fewer non-beta-lactam drugs than HA-MRSA strains and in the USA the strains most commonly associated with these infections (USA 300 and USA 400) frequently carry Panton Valentine Leukocidin ( $p v l)$ genes. Strains producing PVL 
have also often been shown to be avid producers of biofilm, ${ }^{50}$ which may itself may limit the efficacy of antibiotics (see below).

Molecular means (multilocus sequence typing (MLST), pulsed field gel electrophoresis (PFGE) and toxin gene expression) have allowed the differentiation of CA-MRSA from their healthcare-associated (HA)-MRSA counterparts although some MRSA infections with onset in the community have been of the HA-MRSA type. ${ }^{52,53}$ Cases with MRSA are typed for SCCmec, which is the mobile genetic element that carries the mec A gene which codes for methicillin resistance. ${ }^{52} \mathrm{SCCmec}$ is classified into types (based on mec and $c c r$ types) and each type is further classified into subtypes based on differences in the so-called junkyrd (J) region. ${ }^{52}$ HA-MRSA usually carry larger SCCmec types (I, II and III), whereas CA-MRSA are usually smaller (types IV, V, or VII). ${ }^{52}$ The former carry additional beta-lactam resistance genes allowing them to survive more easily in the hospital environment. ${ }^{52}$ PVL genes are more common in CA-MRSA. PVL has a myriad of toxic and pro-inflammatory activities and it appears that these may possibly play a major role in the pathogenesis of CA-MRSA infections, although there is some debate about this issue and other virulence factors may also be important in this respect. ${ }^{52}$

These infections are now recognized as an emerging clinical entity with distinctive clinical features and high morbidity and mortality. ${ }^{52}$ CA-MRSA infections tend to occur more commonly in younger, healthy patients and while they mainly cause skin and soft tissue infections, they may also cause a necrotizing form of pneumonia. ${ }^{11,51,53,54} \mathrm{CA}-$ MRSA infections have become commonplace in the USA, accounting for $>50 \%$ of 
staphylococcal infections in the outpatient setting. ${ }^{51}$ They have also become more common in other parts of the world, although in some areas of the globe they have remained either rare or almost non-existent. ${ }^{50,51,53}$ Classically described is an influenza or influenza-like prodrome, followed by acute shortness of breath, sepsis and hemoptysis. ${ }^{52}$ However, some studies have indicated that these infections do not necessarily follow influenza infections but a number of patients appear to have underlying immunocompromizing conditions (HIV infection, diabetes mellitus, leukaemia, steroid therapy, immunoglobulin disorders). ${ }^{54}$ No clinical features are absolutely characteristic of these infections, but the demonstration of a necrotizing pneumonia on chest radiograph and a rapidly increasing pleural effusion should raise the suspicion. ${ }^{54}$

These infections are more difficult to treat than infections caused by methcillin-sensitive strains of $S$. aureus, since there are relatively few agents available to treat such infections, with some of these agents having significant limitations, and strains have emerged that are resistant to each of the agents that are theoretically available to treat such infections. ${ }^{53}$ Vancomycin or linezolid have been recommended for initial therapy. ${ }^{52}$ However, failures of vancomycin have been documented even with isolates which according to MIC testing were susceptible. ${ }^{52}$ Most studies have documented a high mortality rate and improved outcomes appear to be associated with the use of antimicrobials that inhibit exotoxin production and/or non-toxigenic strains, i.e. clindamycin or linezolid. ${ }^{54}$ It is therefore most commonly recommended that in the case of those strains producing PVL, combination antibiotic therapy is used, with an agent or agents that are able to decrease toxin production, such as linezolid and clindamycin. ${ }^{51,52,54}$ 


\section{Gram-negative rods}

In some areas of the world, gram-negative enteric bacteria, such as Klebsiella pneumoniae, Escherichia coli and various other Enterobacter species appear to be relatively common causes of CAP. ${ }^{4,55}$ Over a number of years there has been a progressive increase in antimicrobial resistance among many of these gram-negative pathogens, including the presence of extended spectrum beta-lactamase (ESBL) production, and although this has been more common among nosocomial infections, cases have also been noted in patients with healthcare-associated pneumonia, as well as those with community-acquired infections, particularly in certain areas of the world. ${ }^{56-59}$ Such infections are known to be associated with an increase in mortality, particularly among patients with pneumonia, and specific therapy, such as with ertapenem, is required for effective treatment. ${ }^{60}$ Various other forms of antimicrobial resistance have been documented in gram-negative pathogens overall. ${ }^{56}$

\section{Alternative mechanisms of microbial resistance}

The following sections will address the roles of microbial biofilm in particular, as well as exposure of bacteria to environmental mutagens or immunosuppression in promoting treatment failure and/or resistance to antibiotics. This is followed by a consideration of non-antibiotic strategies to overcome resistance.

\section{Biofilm}

Encasement in biofilm is a survival strategy used by bacterial pathogens including staphylococcus and streptococcal species, Mycobacterium tuberculosis, E. coli, K. 
pneumoniae, and $P$. aeruginosa, as well as yeasts and fungi such as Candida albicans and Cryptococcus neoformans. ${ }^{61-64}$ Biofilm, which has been implicated in $60-80 \%$ of all microbial infections, ${ }^{65}$ is a hydrated, self-generated, well-ordered matrix of extracellular polymeric substances, mainly polysaccharides, proteins and DNA, with the exception of mycobacterial species, which utilize mycolic acids as a major constitutent of biofilm. ${ }^{63,66}$ When embedded in biofilm, either attached to epithelial surfaces or sequestered intracellularly, microbial pathogens are effectively insulated not only against antibiotics, but also against the cellular and humoral defense mechanisms of the host. In this relatively quiescent state, the bacteria can re-emerge and re-populate previously colonized sites on removal of stressors, or at times when host defenses are transiently compromised as may occur during infection with influenza virus, respiratory syncytial virus or HIV-1. ${ }^{61,62,67}$

Although biofilms may contain a single bacterial strain, most naturally occurring biofilms contain multiple bacterial species and are known as inter-species, multi-species, or polymicrobial biofilms. ${ }^{62,68,69}$ Co-existence in polymicrobial biofilms is not necessarily harmonious, however, due to competition for nutrients, as well as for optimally aerated and irrigated sites. On the other hand, cooperative interactions, especially those which promote resistance to antibiotics, reinforce the overall resilience of the biofilm community. 


\section{Quorum sensing and biofilm formation}

Although the exact molecular mechanisms underpinning biofilm formation remain to be conclusively established, quorum sensing mechanisms activated in response to environmental stress, including antibiotics, and phagocyte-derived reactive oxygen species (ROS) such as hydrogen peroxide, appear to mediate the transition from

planktonic to biofilm growth, ${ }^{62,65,70,71}$ with approximately $4-10 \%$ of the bacterial genome and $\geq 20 \%$ of the proteome being regulated by quorum sensing mechanisms. ${ }^{65}$

Quorum sensing is a process by which microorganisms communicate using chemical messengers known as autoinducers (AIs), usually small soluble molecules or peptides ${ }^{65,72}$ This signalling process enables bacterial communities to coordinate their gene expression according to cell density, promoting the transition to diverse phenotypes on reaching a critical threshold concentration. ${ }^{62}$ Depending on the local microenvironment, quorum sensing systems may upregulate processes which augment virulence, enabling an orchestrated onslaught on the host. Alternatively, as mentioned above in a hostile microenvironment, quorum sensing systems can promote the transition to a less aggressive, persistent state via biofilm formation.

Gram-negative bacteria most commonly utilize type 1 family autoinducers (AI-1) known as N-acylated-L-homoserine lactones (AHLs), of which 3-oxo-hexanoyl-homoserine is the prototype ${ }^{72}$ as their primary inducers of quorum sensing. ${ }^{65,72}$ These are small, soluble, diffusible molecules which vary with respect to their acyl substituents. ${ }^{74}$ They are produced by AHL synthases belonging to the family of LuxI-type proteins. These 
utilize $S$-adenosylmethionine and an acyl-acyl carrier protein to synthesize AHLs, which in turn interact with their cytoplasmic receptors, the LuxR-type proteins, AHL-regulated transcription factors. ${ }^{65,72,75-77}$ Meaningful LuxR-type receptor binding occurs on attainment of a high intracellular concentration of AHLs. The AHL/LuxR-type protein complex usually dimerizes and interacts with the LuxR box in the promoter region of target genes, initiating transcription of quorum sensing controlled genes. ${ }^{65,72,74}$ Over 100 types of gram-negative bacteria utilize Lux1/LuxR-type systems to mediate quorum sensing, with many species having multiple Lux1/LuxR-type pairs which target different and common genes controlled by quorum sensing mechanisms, including those involved in biofilm formation in pathogens such as P. aeruginosa and Burkholderia cepacia complex. $^{65,74,78}$

Gram-positive bacteria, on the other hand, do not produce AHLs, but typically utilize small post-transtationally modified cyclic oligopeptides to initiate quorum sensing. These, in turn, interact with the sensor element of a histidine kinase two-component signal transduction system. ${ }^{65}$ Examples of these are the competence-stimulating peptides and cyclic thiolactone oligopeptides of streptococcal and staphylococcal species respectively, both of which are involved in biofilm formation. ${ }^{65,78,79}$

Autoinducer-2 (AI-2) is a recently described family of quorum sensing molecules utilized by both gram-negative and gram-positive bacteria, apparently to mediate interspecies communication, in contrast to AHLs and cyclic oligopeptides which mediate intraspecies communication. ${ }^{75,80,81}$ The LuxS protein, the product of the widely conserved luxS gene 
found in over 70 species of bacteria, mediates the synthesis of 4,5-dihidroxy-2,3pentadione, the precursor of a pool of AI-2 molecules. ${ }^{75}$ The AI-2-mediated sensing mechanism is well-described in Vibrio harveyi, and is likely to be broadly operative in other types of bacteria. It requires two proteins, LuxP and LuxQ, which are the periplasmic binding and sensor kinase proteins respectively. ${ }^{75}$ These two proteins are thought to form a dimer (LuxP/Q), which is modified following the binding of AI-2 at critical threshold levels to generate LuxR, described by Galloway et al. as "the quorum sensing master regulator that controls expression of the genes in the quorum sensing regulon". 75,82

The involvement of the AI-2 quorum sensing system in biofilm formation has been described in various gram-positive and gram-negative bacterial pathogens including $S$. mutans, S. pneumoniae, S. aureus, S. epidermidis, Enterococcus faecalis, E. coli, $H$. influenzae, Helicobacter pylori, Campylobacter and Salmonella species. ${ }^{83-86}$ Because it promotes bacterial persistence and antibiotic resistance via interspecies communication, the AI-2 quorum sensing system is considered to be a potentially exciting target for treatment of polymicrobial infections. ${ }^{85}$

\section{Biofilm and antibiotic resistance}

Bacterial biofilms provide an environment which is eminently conducive to minimizing or even negating the efficacy of antibiotics, being achieved by both genetic and passive mechanisms. The genetic basis of biofilm-mediated antibiotic resistance involves inter- 
dependent innate and induced resistance mechanisms, ${ }^{87}$ while several mechanisms of passive resistance have been described.

\section{Biofilm-mediated innate resistance}

Notwithstanding the processes involved in formation of biofilm, innate resistance is primarily a function of the mechano-physical properties of biofilm which impede the diffusion of antibiotics, nutrients and oxygen. In the case of antibiotics, the MIC and MBC values of these agents for biofilm-embedded bacteria may be up to 100-1000-fold higher than those of planktonic bacteria, ${ }^{63,88}$ with exposure to low, tolerable concentrations of antibiotics clearly favouring development of genetic drug resistance mechanisms. In the case of nutrients and oxygen, the creation of poorly-aerated, nutrientdepleted zones in the core of the biofilm community results in a slow-down in metabolic activity and growth, resulting in phenotypic resistance. ${ }^{62}$

\section{Biofilm-mediated genetic resistance}

As mentioned above, by retarding the penetration of antibiotics, biofilm-associated innate antibiotic resistance may facilitate the induction of expression of resistance genes. In addition, the close proximity of bacterial cells within the biofilm provides an environment amenable to horizontal gene transfer, which can lead to acquisition of antibiotic resistance. ${ }^{89}$ Indeed inter-strain and inter-species horizontal gene transfer have been demonstrated in biofilms, ${ }^{90,91}$ including transfer of a multi-drug resistance plasmid in E. coli biofilms. ${ }^{77}$ Although ominous, the clinical significance of biofilm-associated 
intra-/inter-strain and inter-species, ${ }^{92}$ horizontal transfer of antibiotic resistance genes remains to be established.

\section{Biofilm-associated passive acquisition of antibiotic resistance}

Passive resistance to $\beta$-lactam antibiotics, unrelated to impaired penetration, has been described for S. pneumoniae in polymicrobial biofilms. Budhani et al., using a continuous culture biofilm system in vitro, reported that a $\beta$-lactam-sensitive strain of the pneumococcus was protected by co-culture with a $\beta$-lactamase-producing strain of $M$. catarrhalis. ${ }^{93}$ Similarly, Weimer et al., using a chinchilla model of biofilm-associated experimental otitis media, demonstrated that a $\beta$-lactamase-producing strain of nontypeable $H$. influenzae also conferred passive protection against amoxicillin on a $\beta$ lactam-sensitive strain of the pneumococcus. ${ }^{94}$

\section{Environmental mutagens and antibiotic resistance}

Although mutagenic in the Ames test, which utilizes auxotrophic strains of Salmonella typhimurium, the potential of environmental mutagens to cause antibiotic resistance by promoting point mutations in bacterial genes encoding proteins which are targets for antibiotics is largely unknown. Very recently, however, Miyahara and colleagues, investigated the effects of exposure of a rifampicin- and ciprofloxacin-sensitive reference strain of $P$. aeruginosa to a series of known mutagens on the development of antibiotic resistance. ${ }^{95}$ The mutagens selected for investigation were: i) the known alkylating agents, ethyl methanesulfonate (EMS) and N-nitroso- $N$-methylurea (MNU); ii) benzopyrene (BP) and 1,6-dinitropyrene (1,6-DNP), both products of combustion; iii) N- 
nitroso-nornicotine (NNN), a constituent of cigarette smoke; and iv) 1,3-bis(2chloroethyl)-1-nitrosurea (BCNU), an anti-cancer agent. All were used at mechanistically (induction of point mutations) or environmentally-relevant concentrations. ${ }^{95}$ The bacteria were exposed to the test mutagens for 24 hours, followed by plating-out and selection of rifampicin- and ciprofloxacin-resistant mutants from which DNA was extracted, amplified by PCR, sequenced and analysed for point mutations in target genes, these being: i) 2-poB, the $\beta$-subunit of RNA polymerase; ii) $g y r A$ and $g y r B$ which encode the A and B proteins of DNA gyrase; iii) par $C$ and par $E$ which encode the A and B subunits of topoisomerase IV; and iv) $n f x B$ and mexR, fluoroquinolone efflux pump regulatory genes.

Exposure of $P$. aeruginosa to EMS, MNU, or BCNU in particular, significantly increased the incidence of resistance to rifampicin, with lesser effects observed with 1,6-DNP, while BP and NNN were relatively ineffective. ${ }^{95}$ Acquisition of resistance was associated with point mutations in about $93 \%$ of the rifampicin-resistant isolates. ${ }^{95}$ Essentially similar effects of these mutagens, albeit at an approximately 10-fold lower frequency, on development of ciprofloxacin resistance were observed, with mutations detected mostly in the $g y r A / B$ and parE genes, but not $\operatorname{par} C, n f x B$ or $\operatorname{mexR} .^{95}$

Although the cigarette smoke-derived mutagen, NNN, was relatively ineffective in promoting antibiotic resistance in $P$. aeruginos $a,{ }^{95}$ this may have resulted from the relatively short exposure of the pathogen to the mutagen, as opposed to the prolonged 
exposure to multiple mutagens in the setting of active, and even passive, cigarette smoking.

In addition, cigarette smoke, and possibly other environmental toxins, have been reported to induce biofilm formation by bacterial pathogens. Exposure of the oral pathogen Porphyromonas gingivalis to cigarette smoke extract in vitro, was found to initiate biofilm formation, ${ }^{96}$ while inhalation of cigarette smoke by humans is associated with the induction of sinonasal microbial biofilms. ${ }^{97}$ In the latter study, Goldstein-Daruech et al. isolated various species of bacteria, including amongst others, S. aureus, S. pneumoniae, K. pneumoniae and P. aeruginosa, from the sinonasal cavities of smokers and nonsmokers. Exposure of the majority (12/16) of bacterial isolates from the sinonasal cavities of smokers to smoke generated by the combustion of 5 reference cigarettes in vitro, followed by incubation for 17 hours, resulted in vigorous biofilm formation. ${ }^{97}$ Similar effects using bacteria isolated from non-smokers necessitated repetitive smoke exposure over a 4 day period. The findings of these two studies ${ }^{96,97}$ are compatible with a role for cigarette smoke-induced biofilm formation in promoting bacterial persistence in the setting of impaired efficacy of antibiotics, which may be compounded by its mutagenic potential.

\section{Immunosuppression and failure of antibiotic therapy}

The critical dependence of cooperative interactions between antibiotics and host defenses in the eradication of microbial pathogens is underscored by the limited efficacy of these agents in patients with primary immunodeficiency disorders, as well as those with 
acquired immunodeficiency. Notwithstanding iatrogenic immunosuppression and HIV infection, old age is one of the most common and increasing causes of acquired immunodeficiency and possible failure of antibiotic therapy, while cigarette smoking interferes with host defenses in the airways.

In the case of the elderly, immunosenescence compounded by multiple comorbid diseases and possibly undernutrition, may interact to complicate the clinical course of infectious diseases, especially pneumonia, urinary tract infections, severe sepsis, meningitis, infective endocarditis, septic arthritis and tuberculosis, often necessitating hospitalization. ${ }^{98-101}$ In the hospital setting, administration of antibiotics, which may be frequent and empiric, predisposes the elderly to the emergence of highly resistant pathogens such as methicillin-resistant S. aureus, $\beta$-lactam-resistant pneumococci, vancomycin-resistant enterococci, and multiple drug-resistant gram-negative bacilli. ${ }^{100,101}$ While not directly promoting antibiotic resistance, age-related immunosuppression may favour its development by contributing to treatment failure.

Cigarette smoking is also a well-documented risk factor for respiratory bacterial infection including tuberculosis ${ }^{102}$ and severe pneumococcal disease, ${ }^{103}$ which is associated with interference with innate and adaptive pulmonary host defenses. In the case of innate immunity, cigarette smoking has been reported to compromise the protective functions of ciliated respiratory epithelium, ${ }^{104}$ and upper airway and tracheobronchial epithelial cells, ${ }^{105,106}$ as well as with the phagocytic activity of pulmonary macrophages. ${ }^{107}$ In an animal model of experimental infection, exposure to cigarette smoke was found to 
interfere with the pulmonary T-cell responses to both M. tuberculosis and influenza virus. ${ }^{108}$

Although unproven, interactions involving mutagenic potential, induction of biofilm formation, and interference with pulmonary host defenses may limit the efficacy of antimicrobial therapy in cigarette smokers, ${ }^{102}$ and possibly contribute to the development of antibiotic resistance. The mechanisms by which smoking may promote treatment failure and resistance are summarized in Table 2.

\section{Non-antibiotic strategies to overcome resistance}

These include implementation of: i) more efficient and extensive immunization programmes targeted at high-risk groups, especially the elderly; ii) improved hygiene, nutrition and social upliftment strategies; and iii) heightened awareness of regional antibiotic resistance frequencies in association with discerning antibiotic prescribing

practices. ${ }^{109}$ In addition, strategies which target biofilm formation and/or its disruption, as well as those which augment innate immunity have the potential to counteract treatment failure and development of antibiotic resistance.

\section{Biofilm-targeted strategies}

Two major strategies have attracted recent attention. These are: i) the design and synthesis of pharmacological agents and monoclonal antibodies which inhibit the synthesis of biofilm-inducing quorum sensors, or which antagonize their receptors on 
bacterial cells; and ii) the identification of non-toxic chemicals and enzymes which promote disruption of biofilm.

\section{Inhibitors of quorum sensing}

As mentioned above, the quorum sensing systems most widely, but not exclusively, utilized by gram-negative bacteria involve the synthesis of AHL and AI-2 autoinducers, ${ }^{65,75}$ both of which converge on LuxR, making this an attractive generic target for development of anti-biofilm strategies. Additional strategies include development of: i) inhibitors of Lux 1-type acyl synthases, as well as LuxS-mediated synthesis of AI-2; ii) antagonists of the interaction of AHL and AI-2 autoinducers with their receptors on Lux R-type and LuxP-type proteins respectively; and iii) strategies which broadly inactivate this family of autoinducers.

Modulation of the AHL and AI-2 quorum sensing pathways by small molecules in gramnegative bacteria has recently been described in considerable detail by Galloway and colleagues. ${ }^{75}$ These include analogues of AHLs and $S$-adenosylmethionine, which target type $1 \mathrm{AI}$, as well as cinnamaldehyde and furanones which target both types of $\mathrm{AI}$ and are potentially useful in eradicating polymicrobial biofilms containing gram-negative and gram-positive bacteria. ${ }^{65,75,85,110,111}$ Other types of compound have also been reported to inhibit biofilm formation by undefined, possibly antimicrobial, mechanisms. These include aryl 2-aminoimidazoles and their derivatives in the case of gram-negative bacteria, ${ }^{112,113}$ and 3-arylideneflavonones which alter the integrity of the outer membrane in gram-positive bacteria, possibly antagonizing the uptake of AIs, ${ }^{114}$ and pyrroloindoline triazole amides, which appear to antagonize indole metabolism. ${ }^{115}$ Indole functions as an 
intracellular signal regulating various phenotypes in both gram-negative and grampositive bacteria, including biofilm formation. ${ }^{115}$ Hamamelitannin, an anti-oxidant derived from witch hazel, has been found to antagonize the effects of the cylic thiolactone autoinducing peptide of S. aureus on biofilm formation, but toxicity and lack of selectivity may restrict its therapeutic potential. ${ }^{116}$

Although most of these small molecules have demonstrated efficacy in both in vitro and in vivo models of experimental biofilm infection, usually in combination with antibiotics, ${ }^{79}$ their efficacy in the clinical setting remains unproven. Nonetheless the approach is innovative and promising. ${ }^{75}$

Monoclonal antibodies which target the cyclic oligopeptide AIs of Gram-positive bacteria also have the potential to antagonize biofilm formation, and have indeed demonstrated efficacy in a murine model of experimental infection with S. aureus. ${ }^{117}$ In the clinical setting, however, the therapeutic application of anti-biofilm monoclonal antibodies may be restricted due to issues such as expense, susceptibility to proteolytic cleavage, poor penetration into biofilms, and selectivity for a single, as opposed to a range, of AIs. On a more positive note, however, crystallographic analysis of monoclonal antibody/cyclic oligopeptide AI interactions may reveal structural interactions which would facilitate the development of broadly cross-reactive antibodies or vaccines, at least in the case of $S$. aureus. ${ }^{117}$ 


\section{Biofilm-disrupting strategies}

These are generally based on the use of enzymes which cleave the major components of biofilm (polysaccharidases, proteinases, nucleases), as well as oxidizing agents and surfactants/detergents. ${ }^{118,119}$ Because of the multi-constituent nature of biofilm, which varies considerably between species, these agents are generally used in combination. However, their lack of selectivity, together with their pro-inflammatory and pro-allergic potential, underpins their primary use as disinfectants as opposed to therapeutic agents. A notable exception is the use of nebulized DNAase for prevention of $P$. aeruginosa biofilms in the airways of patients with cystic fibrosis. ${ }^{59}$

\section{Potentiation of innate immunity}

Although largely untested in the therapeutic setting in humans, augmentation of innate immunity has been identified as a strategy to increase the efficacy of antimicrobial chemotherapy and possibly counter antibiotic resistance. ${ }^{120-122}$ Two such strategies involve the use of inhibitors of cyclooxygenase 1 (COX1) or antagonists of Toll-like receptors (TLRs).

\section{Inhibitors of $C O X 1$}

COX inhibitors effectively attenuate the production of 3'-5' -cyclic adenosine monophosphate (cAMP) by cells of the innate immune system. They do so by inhibiting the production of prostaglandins ( $\mathrm{PGs}) \mathrm{E}_{2}$ and $\mathrm{D}_{2}$, attenuating their autocrine interaction with adenylyl cyclase-activating EP2/4 and DP1 receptors on phagocytic cells. Cyclic 
AMP, which has been described as the "master regulator of innate immune cell function," ${ }^{123}$ is a potent inhibitor of Fc $\gamma \mathrm{R}$-mediated phagocytosis, as well as production of antimicrobial ROS, and release of antimicrobial peptides/proteins. ${ }^{123}$

Stables et al., using a murine model of experimental group B Streptococcus-induced peritonitis, reported that intraperitoneal administration of inhibitors of COX1, but not $\mathrm{COX} 2$, as well as an antagonist of EP2/4 and DP1 receptors, either 1 hour pre- or postinduction of experimental infection, was associated with decreased bacterial survival in the setting of increased phagocytic activity of isolated peritoneal leukocytes, as well as enhanced production of ROS. ${ }^{120}$ Similarly, oral administration of a single $500 \mathrm{mg}$ tablet of naproxen to adult human volunteers, followed one hour later by ex vivo determination of killing of a penicillin-sensitive strain of $S$. pneumoniae, using a whole blood assay, revealed increased phagocytic activity, generation of ROS and antimicrobial activity. ${ }^{120}$ Similar effects on antimicrobial activity were observed using a penicillin-resistant strain of $S$. pneumoniae, although the effects of combinations of penicillin and COX1 inhibitors were not tested. ${ }^{120}$ These authors advocate "the potential of inhibitors of PG signalling pathways as adjunctive therapies, particularly in the context of antibiotic resistance." 120 On a more cautious note, however, the augmentative effects of agents which interfere with cAMP-mediated control of inflammatory responses may predispose to inflammation-mediated tissue damage and organ dysfunction. 


\section{Agonists of Toll-like receptors}

Agonists of TLR2 and TLR4 in particular may be useful in potentiating innate immunity by increasing the synthesis of pro-inflammatory cytokines, especially interleukin(IL)-1, IL-6, IL-8 and TNF by cells of the innate immune system, as well as by epithelial cells. ${ }^{121,122}$ However, the use of this type of immunomodulatory strategy, as well as the direct administration of recombinant cytokines such as interferon- $\gamma$ and granulocyte colony-stimulating factor, or host-derived antimicrobial peptides is likely to be complicated by an inability to selectively target the microbial pathogen. In this setting, inappropriate inflammatory responses are likely to be potentially damaging as opposed to protective.

\section{Conclusion}

This review highlights many of the issues associated with antimicrobial resistance and antibiotic treatment failure. Both of these can only be countered using a combination of strategies. Clearly, foremost are the development of novel antimicrobial agents and more discerning use of existing agents. Others strategies include disease prevention targeting high-risk groups, particularly the elderly, more aggressive and effective anti-smoking campaigns, and development of pharmacological and immunological adjuvants which complement antibiotics, in particular those which target biofilm. It is reassuring that the threat posed by biofilm infections is well-recognized, and that innovation, particularly in the development field of small molecule antagonists of biofilm formation, show considerable promise. 


\section{References}

1. Welte T, Torres A, Nathwani D. Clinical and economic burden of communityacquired pneumonia among adults in Europe. Thorax 2010 doi: 10.1136/thx.2009.129502.

2. File TM Jr., Marrie TJ. Burden of community-acquired pneumonia in North American adults. Postgraduate Medicine 2010; 122(2): 130-41.

3. Isturiz RE, Luna CM, Ramirez J. Clinical and economic burden of pneumonia among adults in Latin America. Int J Infect Dis 2010; 14: e852 - e856

4. Song J-H, Thamlikitkul V, Hsueh P-R. Clinical and economic burden of communityacquired pneumonia amongst adults in the Asia-Pacific region. Int J Antimicrob Agents 2011; 38: $108-17$.

5. Ramirez JA, Anzueto AR. Changing needs of community-acquired pneumonia. J Antimicrob Chemother 2011; 66 (suppl 3): iii3 - iii9.

6. Huang SS, Johnson KM, Ray GT, et al. Healthcare utilization and cost of pneumococcal disease in the United States. Vaccine 2011; 29: $3398-412$.

7. Ewig S, Torres A. Community-acquired pneumonia as an emergency: time for an aggressive intervention to lower mortality. Eur Respir J 2011; 38: 253 - 60 .

8. Feikin DR, Feldman C, Schuchat A, Janoff EN. Global strategies to prevent bacterial pneumonia in adults with HIV disease. Lancet Infect Dis 2004; 4: $445-55$.

9. Welte T, Köhnlein T. Global and local epidemiology of community-acquired pneumonia: The experience of the CAPNETZ Network. Seminars Respir Crit Care Med 2009; 30: $127-35$. 
10. Woodhead M. The European vision of community-acquired pneumonia. Seminars Respir Crit Care Med 2009; 30: 136 - 45.

11. Niederman MS. Community-acquired pneumonia: The U.S. perspective. Seminars Respir Crit Care Med 2009; 30: $179-88$.

12. Jones RN, Jacobs MR, Sader HS. Evolving trends in Streptococcus pneumoniae resistance: implications for therapy of community-acquired pneumonia. Int $\mathbf{J}$ Antimicrob Agent 2010; 36: $197-204$.

13. Polverino E, Marti AT. Community-acquired pneumonia. Minerva Anestesiologica 2011; 77: $196-211$.

14. Metlay JP, Singer DE. Outcomes in lower respiratory tract infections and the impact of antimicrobial drug resistance. Clin Microbiol Infect 2002; 8 (suppl 2): $1-11$.

15. Jacobs MR, Anon J, Appelbaum PC. Mechanisms of resistance among respiratory tract pathogens. Clin Lab Med 2004; 24: 419 - 53.

16. Lynch JP III, Zhanel GG. Streptococcus pneumoniae: epidemiology and risk factors, evolution of antimicrobial resistance, and impact of vaccines. Curr Opin Pulm Med 2010; 16: $217-25$.

17. Feldman C. Clinical relevance of antimicrobial resistance in the management of pneumococcal community-acquired pneumonia. J Lab Clin Med 2004; 143: 269 83.

18. Lynch JP, III, Zhanel GG. Streptococcus pneumoniae: Does antimicrobial resistance matter? Seminars Respir Crit Care Med 2009; 30: 210 - 38.

19. Feldman C, Anderson R. Bacteraemic pneumococcal pneumonia. Drugs 2011; 71(2): $131-53$. 
20. Felmingham D, Feldman C, Hryniewicz W, et al. Surveillance of resistance in bacteria causing community-acquired respiratory tract infections. Clin Microbiol Infect 2002; 8 (supp1 2): $12-42$.

21. Klugman KP, Low DE, Metlay J, Pechere J-C, Weiss K. Community-acquired pneumonia: new management strategies for evolving pathogens and antimicrobial susceptibilities. Int J Antimicrob Agents 2004; 24: 411 - 22.

22. Alpuche C, Garau J, Lim V. Global and local variations in antimicrobial susceptibilities and resistance development in the major respiratory pathogens. Int $\mathrm{J}$ Antimicrob Agents 2007; 30S: S135-S138.

23. Goto H, Shimada K, Ikemoto H, Oguri T, the Study Group on antimicrobial susceptibility of Pathogens Isolated from Respiratory Infections. Antimicrobial susceptibility of pathogens isolated from more than 10000 patients with infectious respiratory diseases: a 25-year longitudinal study. J Infect Chemother 2009; 15: $347-60$.

24. File TM, Jr. The science of selecting antimicrobials for community-acquired pneumonia (CAP). JMCP 2009; 15(2)(suppl): S5 - S11.

25. Jacobs MR. Optimisation of antimicrobial therapy using pharmacokinetic and pharmacodynamic parameters. Clin Microbiol Infect 2001; 7: $589-96$.

26. Garau J. Role of beta-lactam agents in the treatment of community-acquired pneumonia. Eur J Clin Microbiol Infect Dis 2005; 24: 83 - 99.

27. Owens RC, Jr., Shorr AF. Rational dosing of antimicrobial agents: Pharmacokinetic and pharmacodynamic strategies. Am J Health-Syst Pharm 2009; 66 (suppl 4): S23 $-\mathrm{S} 30$. 
28. Van Bambeke F, Reinert RR, Appelbaum PC, Tulkens PM, Peetermans WE. Multidrug-resistant Streptococcus pneumoniae infections. Current and future therapeutic options. Drugs 2007; 67(16): $2355-82$.

29. Jacobs MR. Antimicrobial-resistant Streptococcus pneumoniae: trends and management. Expert Rev Anti Infect Ther 2008; 6(5): $619-35$.

30. Song J-H, Chung DR. Respiratory infections due to drug-resistant bacteria. Infect Dis Clin N Am 2010; 24: 639 - 53.

31. Feldman C, Brink AJ, von Gottberg A, et al. Antimicrobial susceptibility of pneumococcal isolates causing bacteraemic pneumococcal pneumonia: analysis using current breakpoints and fluoroquinolone pharmacodynamics. Int J Antimicrob Agents 2010; 36: $95-7$.

32. Yu VL. Chiou CC, Feldman C, et al; International Pneumococcal Study Group. An international prospective study of pneumococcal bacteremia: correlation with in vitro resistance, antibiotics administered, and clinical outcome. Clin Infect Dis 2003; 37 (2): $230-7$.

33. Amsden GW. Pneumococcal macrolide resistance - myth or reality? J Antimicrob Chemother 1999; 44: 1- 6 .

34. Lonks JR, Garau J, Medeiros AA. Implications of antimicrobial resistance in the empirical treatment of community-acquired respiratory tract infections: the case of macrolides. J Antimicrob Chemother 2002; 50: (suppl S2): 87 - 91 .

35. Lynch JP III, Martinez FJ. Clinical relevance of macrolide-resistance Streptococcus pneumoniae for community-acquired pneumonia. Clin Infect Dis 2002; 34 (suppl 1): S27 - S46. 
36. Nuermberger E, Bishai WR. The clinical significance of macrolide-resistant Streptococcus pneumoniae: It's all relative. Clin Infect Dis 2004; 38: 99 - 103.

37. Fuller JD, McGeer A, Low DE. Drug-resistant pneumococcal pneumonia: clinical relevance and approach to management. Eur J Clin Microbiol Infect Dis 2005; 24: $780-88$

38. Pletz MW, van der Linden M, von Baum H, Duesberg CB, Klugman KP, Welte T, for the CAPNETZ study group. Low prevalence of fluoroquinolone resistant strains and resistance precursor strains in Streptococcus pneumoniae from patients with community-acquired pneumonia despite high fluoroquinolone usage. International Journal of Medical Microbiology 2011; 301: $53-7$.

39. Lismond A, Carbonnelle S, Tulkens PM, van Bambeke F. Efflux of novel quinolones in contemporary Streptococcus pneumoniae isolates from community-acquired pneumonia. J Antimicrob Chemother 2011; 10: $948-51$.

40. Fuller JD, Low DE. A review of Streptococcus pneumoniae infection treatment failures associated with fluoroquinolone resistance. Clin Infect Dis 2005; 41: $118-$ 21.

41. Arnold FW, Summersgill JT, Lajoie AS, et al., and the Community-Acquired Pneumonia Organization (CAPO) investigators. A worldwide perspective of atypical pathogens in community-acquired pneumonia. Am J Respir Crit Care Med 2007; 175: $1086-93$.

42. Isozumi R, Yoshimine H, Morozumi M, Ubukata K, Ariyoshi K. Adult communityacquired pneumonia caused by macrolide resistant Mycoplasma pneumoniae. Respirology 2009; 14: $1206-16$. 
43. Morozumi M, Takahashi T, Ubukata K. Macrolide-resistant Mycoplasma pneumoniae: characteristics of isolates and clinical aspects of community-acquired pneumonia. J Infect Chemother 2010; 16: $78-86$.

44. Miyashita N, Maruyama T, Kobayashi T, et al. Community-acquired macrolideresistant Mycoplasma pneumoniae pneumonia in patients more than 18 years of age. J Infect Chemother 2011; 17: $114-8$.

45. Bẹ́bẹ́ar C, Pereyre S, Peuchant O. Mycoplasma pneumoniae: Susceptibility and resistance to antibiotics. Future Microbiology 2011; 6(4): 423 - 31 .

46. Hoban D, Felmingham D. The PROTEKT surveillance study: antimicrobial susceptibility of Haemophilus influenzae and Moraxella catarrhalis from communityacquired respiratory tract infections. J Antimicrob Chemother 2002; 50 (suppl S1): $49-59$.

47. Tristram S, Jacobs MR, Appelbaum PC. Antimicrobial resistance in Haemophilus influenzae. Clin Microbiol Rev 2007; 20: $368-89$.

48. McGregor K, Chang BJ, Mee BJ, Riley TV. Moraxella catarrhalis: Clinical significance, antimicrobial susceptibility and BRO beta-lactamases. Eur J Clin Microbiol Infect Dis 1998; 17: $219-34$.

49. Murphy TF, Parameswaran GI. Moraxella catarrhalis, a human respiratory tract pathogen. Clin Infect Dis 2009; 49: $124-31$.

50. Stefani S, Bongiorno D, Cafiso V, et al. Pathotype and susceptibility profile of a community-acquired methicillin-resistant Staphylococcus aureus strain responsible for a case of severe pneumonia. Diagn Microbiol Infect Dis 2009; 63(1): $100-4$. 
51. Cataldo MA, Taglietti F, Petrosillo N. Methicillin-resistant Staphylococcus aureus: a community health threat. Postgrad Med 2010;122(6): $16-23$.

52. Hidron AI, Low CE, Honig EG, Blumberg HM. Emergence of community-acquired methicillin-resistant Staphylococcus aureus strain USA300 as a cause of necrotizing community-onset pneumonia. Lancet Infect Dis 2009; 9: $384-92$.

53. David MZ, Daum RS. Community-associated methicillin-resistant Staphylococcus aureus: epidemiology and clinical consequences of an emerging epidemic. Clin Microbiol Rev 2010; 23 (3): $616-87$.

54. Lobo LJ, Reed KD, Wunderink RG. Expanded clinical presentation of communityacquired methicillin-resistant Staphylococcus aureus pneumonia. Chest 2010; 138(1): $130-6$.

55. Marques MR, Nunes A, Sousa C, et al. Community-acquired pneumonia in an intensive care unit. Rev Port Pneumol 2010; 16(2): 223 - 35.

56. Sader HS, Sampaio JL, Zocoli C, Jones RN. Results of the 1997 SENTRY Antimicrobial surveillance program in three Brazilian medical centres. Braz J Infect Dis 1999; $3(2): 63-79$.

57. Einhorn AE, Neuhauser MM, Bearden DT, et al. Extended-spectrum betalactamases: frequency, risk factors, and outcomes. Pharmacotherapy 2002; 22(1): $14-20$.

58. Kang C-I, Song J-H, Chung DR, et al., on behalf of the Korean Network for Study of Infectious diseases (KONSID). Risk factors and treatment outcomes of communityonset bacteraemia caused by extended-spectrum $\beta$-lactamase-producing Escherichia coli. Int J Antimicrob Agents 2010; 36: $284-87$. 
59. Tsai SS, Huang JC, Chen ST, et al. Characteristics of Klebsiella pneumoniae bacteremia in community-acquired and nosocomial infections in diabetic patients. Chang Gung Med J 2010; 33(5): $532-9$.

60. Teng CP, Chen HH, Chan J, Lye DCB. Ertapenem for the treatment of extendedspectrum $\beta$-lactamase-producing gram-negative bacterial infections. Int J Antimicrob Agents 2007; 30: $356-9$.

61. Costerton JW, Stewart PS, Greenberg EP. Bacterial biofilms: a common cause of persistent infections. Science 1999; 284: $1318-22$.

62. Hall-Stoodley L, Stoodley P. Evolving concepts in biofilm infections. Cell Microbiol 2009; 11: $1034-43$.

63. Ojha AK, Baughn AD, Sambandan D, Hsu T, Trivelli X, Guerardel Y, et al. Growth of Mycobacterium tuberculosis biofilms containing free mycolic acids and harbouring drug-tolerant bacteria. Mol Microbiol 2008; 69: $164-74$.

64. Williams DW, Kuriyama T, Silva S, Malic S, Lewis MAO. Candida biofilms and oral candidosis: treatment and prevention. Periodontol 2000, 2011; 55: $250-65$.

65. Deep A, Chaudhary U, Gupta V. Quorum sensing and bacterial pathogenicity: From molecules to disease. J Lab Physicians 2011; 3: 4-11.

66. Nguyen KT, Piastro K, Gray TA, Derbyshire KM. Mycobacterial biofilms facilitate horizontal DNA transfer between strains of Mycobacterium smegmatis. J Bacteriol 2010; 192: $5134-42$.

67. Anderson R, Feldman C. Key virulence factors of Streptococcus pneumoniae and non-typeable Haemophilus influenzae: roles in host defence and immunization. SA J Epidemiol Infect 2011; 26: 6-12. 
68. Federle MJ, Bassler BL. Interspecies communication in bacteria. J Clin Invest 2003; 112: $1291-99$.

69. Yang L, Liu Y, Wu H, Høiby N, Molin S, Song Z-j. Current understanding of multispecies biofilms. Int J Oral Sci 2011; 3: $74-81$.

70. Elliott D, Burns JL, Hoffman LR. Exploratory study of the prevalence and clinical significance of tobramycin-mediated biofilm induction in Pseudomonas aeruginosa isolates from cystic fibrosis patients. Antimicrob Agents Chemother 2010; 54: 3024 $-6$.

71. Mathee K, Ciofu O, Sternberg C, Lindum PW, Campbell JIA, Jensen P, et al. Mucoid conversion of Pseudomonas aeruginosa by hydrogen peroxide: a mechanism for virulence activation in the cystic fibrosis lung. Microbiol 1999; 145: $1349-57$.

72. McInnis CE, Blackwell HE. Thiolactone modulators of quorum sensing revealed through library design and screening. Biorg Med Chem 2011; 19: 4820 - 28.

73. Eberhard A, Burlingame AL, Eberhard C, Kenyon GL, Nealson KH, Oppenheimer NJ. Structural identification of autoinducer of Photobacterium fischeri luciferase. Biochemistry 1981; 20: $2444-9$.

74. Fuqua C, Greenberg EP. Listening in on bacteria: acyl-homoserine lactone signaling. Nat Rev Mol Cell Biol 2002; 3: 685 - 95.

75. Galloway WRJD, Hodgkinson JT, Bowden SD, Welch M, Spring DR. Quorum sensing in Gram-negative bacteria: small molecule modulation of the AHL and AI-2 quorum sensing pathways. Chem Rev 2011; 111: 28 - 67 . 
76. Zeng L-R, Xie J-P. Molecular basis underlying LuxR family transcription factors and function diversity and implications for novel antibiotic drug targets. J Cell Biochem 2011 (DOI 10.1002/jcb.23262).

77. Patankar AV, Gonzalez JE. Orphan LuxR regulators of quorum sensing. FEMS Microbiol Rev 2009; 33: $739-56$.

78. Senadheera D, Cvitkovitch DG. Quorum sensing and biofilm formation by Streptococcus mutans. Adv Exp Biol Med 2008; 631: 178 - 88.

79. Brackman G, Cos P, Maes L, Nelis HJ, Coenye T. Quorum sensing inhibitors increase the susceptibility of bacterial biofilms to antibiotics in vitro and in vivo. Antimicrob Agents Chemother 2011; 55: $2655-61$.

80. Ng WL, Bassler BL. Bacterial quorum-sensing network architectures. Annu Rev Genet 2009; 43: $197-222$.

81. Xavier KB, Bassler BL. Interference with AI-2-mediated bacterial cell-cell communication. Nature 2005; 437: $750-3$.

82. Neiditch MB, Federle MJ, Miller ST, Bassler BL, Hughson FM. Regulation of LuxPQ receptor activity by the quorum-sensing signal autoinducer-2. Mol Cell 2005; 18 : $507-18$.

83. Yoshida A, Ansai T, Takehara T, Kuramitsu H. LuxS-based signaling affects Streptococcus mutans biofilm formation. Appl Environ Microbiol 2005; 71: 2372 80.

84. Vidal JE, Ludewick HP, Kunkel RM, Zähner D, Klugman KP. The LuxS-dependent quorum sensing system regulates early biofilm formation by Streptococcus pneumoniae strain D39. Infect Immun 2011 [E pub ahead of print]. 
85. Armbruster CE, Swords WE. Interspecies bacterial communication as a target for therapy in otitis media. Expert Rev Anti Infect Ther 2010; 8: 1067 - 70.

86. Cloak OM, Solow BT, Briggs CE, Chen C-Y, Fratamico PM. Quorum sensing and production of autoinducer-2 in Campylobacter spp., Escherichia coli O157:H7, and Salmonella enterica serovar Typhimurium in foods. Appl Environ Microbiol 2002; 68: $4666-71$.

87. Anderson GG, O’Toole GA. Innate and induced resistance mechanisms of bacterial biofilms. Curr Top Microbiol Immunol 2008; 322: 85 - 105.

88. Høiby N, Bjarnsholt T, Givskov M, Molin S, Ciofu O. Antibiotic resistance of bacterial biofilms. Int J Antimicrob Agents 2010; 35: $322-32$.

89. Roberts AP, Mullany P. Oral biofilms: a reservoir of transferable, bacterial, antimicrobial resistance. Expert Rev Anti Infect Ther 2010; 8: 1441 - 50.

90. Antonova ES, Hammer BK. Quorum-sensing autoinducer molecules produced by members of a multispecies biofilm promote horizontal gene transfer to Vibrio cholerae. FEMS Microbiol Lett 2011; 322: $68-76$.

91. Król JE, Nguyen HD, Rogers LM, Beyenal H, Krone SM, Top EM. Increased transfer of a multi-drug resistance plasmid in E. coli biofilms at the air-liquid interface. Appl Environ Microbiol 2011 [Epub ahead of print].

92. Vaidya VK. Horizontal transfer of antimicrobial resistance by extended spectrum $\beta$ lactamase-producing Enterobacteriaceae. J Lab Physicians 2011; 3: 37 - 42.

93. Budhani RK, Struthers JK. Interaction of Streptococcus pneumoniae and Moraxella catarrhalis: investigation of the indirect pathogenic role of beta-lactamase-producing 
moraxellae by use of a continuous culture biofilm system. Antimicrob Agents Chemother 1998; 42: $2521-26$.

94. Weimer KE, Juneau RA, Murrah KA, Pang B, Armbruster CE, Richardson SH, et al. Divergent mechanisms for passive pneumococcal resistance to $\beta$-lactam antibiotics in the presence of Haemophilus influenzae. J Infect Dis 2011; 203: 549 - 55 .

95. Miyahara E, Nishie M, Takumi S, Miyanohara H, Nishi J, Yoshiie K, et al. Environmental mutagens may be implicated in the emergence of drug-resistant microorganisms. FEMS Microbiol Lett 2011; 317: $109-16$.

96. Bagaitkar J, Demuth DR, Daep CA, Renaud DE, Pierce DL, Scott DA. Tobacco upregulates $P$. gingivalis fimbrial proteins which induce TLR2 hyposensitivity. PLOS ONE 2010; 5: e9323.

97. Goldstein-Daruech N, Cope EK, Zhao K-Q, Vukovic K, Kofonow JM, Doghramji L, et al. Tobacco smoke mediated induction of sinonasal microbial biofilms. PLOS ONE 2011; 6: e15700.

98. Ginaldi L, Loreto MF, Pia Corsi M, Modesti M, De Martinis M. Immunosenescence and infectious diseases. Microbe Infect 2001; 3: $851-57$.

99. McElhaney JE, Effros RB. Immunosenescence: What does it mean to health outcomes in older adults? Curr Opin Immunol 2009; 21: 418 - 24.

100. Yoshikawa TT. Antimicrobial resistance and aging: beginning of the end of the antibiotic era? J Am Geriatr Soc 2002; 50: S226-9.

101. Girard TD, Opal SM, Ely EW. Insights into severe sepsis in older patients: from epidemiology to evidence-based management. Clin Infect Dis 2005; 40: $719-727$. 
102. Tachfounti N, Nejjari C, Benjelloun MC, Berraho M, Elfakir S, El Rhazi K, et al. Association between smoking status, other factors and tuberculosis treatment failure in Morocco. Int J Tuberc Lung Dis 2011; 15: $838-43$.

103. Nuorti JP, Butler JC, Farley MM, Harrison LH, McGeer A, Kolczak MS, et al. Cigarette smoking and invasive pneumococcal disease. N Engl J Med 2000; 342: $681-9$.

104. Stanley PJ, Wilson R, Greenstone MA, MacWilliam L, Cole PJ. Effect of cigarette smoking on nasal mucociliary clearance and ciliary beat frequency. Thorax $1986 ; 41: 519-23$.

105. Manzel LJ, Shi L, O’Shaughnessy PT, Thorne PS, Look DC. Inhibition by cigarette smoke of nuclear factor-кB-dependent response to bacteria in the airway. Am J Respir Cell Mol Biol 2011; 44: 155 - 65.

106. Kulkarni R, Rampersaud R, Aguilar JL, Randis TM, Kreindler JL, Ratner AJ. Cigarette smoke inhibits airway epithelial cell innate immune responses to bacteria. Infect Immun 2010; 78: $2146-52$.

107. Phipps JC, Aronoff DM, Curtis JL, Goel D, O’Brien E, Mancuso P. Cigarette smoke exposure impairs pulmonary bacterial clearance and alveolar macrophage complement-mediated phagocytosis of Streptococcus pneumoniae. Infect Immun 2010; 78: $1214-20$.

108. Feng Y, Kong Y, Barnes PF, Huang FF, Klucar P, Wang X, et al. Exposure to cigarette smoke inhibits the pulmonary T-cell response to influenza virus and Mycobacterium tuberculosis. Infect Immun 2011; 79: 229 - 37. 
109. Zhang Y. Mechanisms of antibiotic resistance in the microbial world. www.moleculartb.org/gb/pdf/transcriptions/11_YZHANG.pdf

110. Lönn-Stensrudd J, Landin MA, Benneche T, Petersen FC, Scheie AA. Furanones, potential agents for preventing Staphylococcus epidermidis biofilm infections? J Antimicrob Chemother 2009; 63: $309-16$.

111. Brackman G, Celen S, Hillaert U, Van Calenbergh S, Cos P, Maes L, et al. Structure-activity relationship of cinnamaldehyde analogs as inhibitors of AI-2 based quorum sensing and their effect on virulence of Vibrio spp. PLOS ONE 2011; 6: e16084.

112. Bunders CA, Richards JJ, Melander C. Identification of aryl 2-aminoimidazoles as biofilm inhibitors in Gram-negative bacteria. Bioorg Med Chem Lett 2010;20: $3797-800$.

113. Steenackers HP, Ermolat'ev DS, Savaliya B, Weerdt AD, Coster DD, Shah A, et al. Structure-activity relationship of 2-hydroxy-2-aryl-2,3-dihydro-imidazo[1,2a]pyrimidinium salts and 2N-substituted 4(5)-aryl-2-amino-1H-imidazoles as inhibitors of biofilm formation by Salmonella typhimurium and Pseudomonas aeruginosa. Biorg Med Chem 2011; 19: $3462-73$.

114. Budzyńska A, Rōzalski M, Karolczak W, Wieckowska-Szakiel M, Sadowska B, Rōzalska B. Synthetic 3-arylideneflavanones as inhibitors of the initial stages of biofilm formation by Staphylococcus aureus and Enterococcus faecalis. Z. Naturforsch C 2011; 66: 104 - 14. 
115. Bunders C, Cavanagh J, Melander C. Flustramine inspired synthesis and biological evaluation of pyrroloindoline triazole amides as novel inhibitors of bacterial biofilms. Org Biomol Chem 2011; 9: 5476 - 81 .

116. Sintim HO, Smith JAJ, Wang J, Nakayama S, Yan L. Paradigm shift in discovering next-generation anti-infective agents: targeting quorum sensing, c-diGMP signaling and biofilm formation in bacteria with small molecules. Future Med Chem 2010; 2: $1005-35$.

117. Kirchdoerfer RN, Garner AL, Flack CE, Mee JM, Horswill AR, Janda KD, et al. Structural basis for ligand recognition and discrimination of a quorum-quenching antibody. J Biol Chem 2011; 286: $17351-58$.

118. Chaignon P, Sadovskaya I, Ragunah Ch, Ramasubbu N, Kaplan JB, Jabbouri S. Susceptibility of staphylococcal biofilms to enzymatic treatments depends on their chemical composition. Appl Microbiol Biotechnol 2007; 75: 125 - 32 .

119. Høiby N, Ciofu O, Johansen HK, Song Z-j, Moser C, Jensen PØ, et al. The clinical impact of bacterial biofilms. Int J Oral Sci 2011; 3: $55-65$.

120. Stables MJ, Newson J, Ayoub SS, Brown J, Hyams CJ, Gilroy DW. Priming innate immune responses to infection by cyclooxygenase inhibition kills antibiotic susceptible and resistant bacteria. Blood 2010;116: $2950-9$.

121. O’Neill LAJ, Bryant CE, Doyle SL. Therapeutic targeting of Toll-like receptors for infectious and inflammatory diseases and cancer. Pharm Rev 2009; 61: 177 - 97 . 122. Nicholls EF, Madera L, Hancock REW. Immunomodulators as adjuvants for vaccines and antimicrobial therapy. Ann NY Acad Sci 2010; 1213: 46 - 61 . 
123. Serezani CH, Ballinger MN, Aronoff DM, Peters-Golden M. Cyclic AMP: master regulator of innate immune cell function. Am J Respir Cell Mol Biol 2008; 39: $127-32$. 
Table 1: Antibiotic resistance among common respiratory pathogens causing community-acquired pneumonia*

\begin{tabular}{|c|c|c|}
\hline Microorganism & $\underline{\text { Antibiotic class }}$ & Common mechanisms of resistance \\
\hline \multirow[t]{3}{*}{ Streptococcus pneumoniae } & beta - lactam & - alterations in penicillin-binding proteins (PBP) \\
\hline & macrolide & $\begin{array}{l}\text { - efflux pump (mef A gene) } \\
\text { - ribosomal merthylation mechanism (erm B gene) }\end{array}$ \\
\hline & fluoroquinolone & $\begin{array}{l}\text { - mutations in fluoroquinolone resistance } \\
\text { determining regions (par C and gyr A) } \\
\text { - (efflux pump) }\end{array}$ \\
\hline Mycoplasma pneumoniae & macrolide & - point mutation in domain $\mathrm{V}$ of the $23 \mathrm{~S}$ rRNA gene \\
\hline Haemophilus influenzae & beta-lactamase & $\begin{array}{l}\text { - beta-lactamase production } \\
\text { (TEM-1, ROB- } 1 \text {, and poorly defined other) } \\
\text { - (alteration of PBP) } \\
\text { - (macrolide efflux pump) }\end{array}$ \\
\hline Moraxella catarrhalis & beta-lactamase & $\begin{array}{l}\text { - beta-lactamase production } \\
\text { (BRO-1, BRO-2, BRO-3) }\end{array}$ \\
\hline Staphylococcus aureus & "methicillin" & - mec A gene \\
\hline Gram negative rods & various & $\begin{array}{l}\text { - beta-lactamase production, including extended } \\
\text { spectrum beta-lactamase (ESBL) } \\
\text { - various other mechanisms }\end{array}$ \\
\hline
\end{tabular}

*Common mechanisms of resistance are highlighted and some of the less common mechanisms are indicated in brackets 
Table 2: Mechanisms by which cigarette smoking may predispose to antibiotic treatment failure and resistance

\section{$\underline{\text { Mechanism }}$}

- Mutagenic activity

- Induction of biofilm formation

- Immunosuppression

\section{$\underline{\text { Effect }}$}

Mutations in antibiotic target genes ${ }^{95}$

Impaired access of antibiotics to target pathogens $^{96,97}$

Dysfunction of the mucociliary escalator; $^{104}$

Impaired cytokine secretion by airway epithelial cells; ${ }^{105,106}$

Decreased phagocytic activity of alveolar macrophages; ${ }^{107}$

Impaired activation of T-cells by airway pathogens. ${ }^{108}$ 